\title{
Fragmentación de pastizales en el centro de la provincia de Buenos Aires, mediante imágenes LANDSAT
}

\author{
Bruno Daniel Lara*
}

Recibido el 21 de diciembre de 2015; aceptado el 5 de junio de 2016

\begin{abstract}
In the current work, a combination of land-cover dynamics maps and landscape pattern metrics based on remote sensing images was employed to quantify the spatial and temporal land cover changes and fragmentation patterns in the centre of the Province of Buenos Aires (Argentina), in the period 1974-2011. This study area covers two agro-ecological zones: the Flooding Pampa and the Inland Pampa. In contrast with other regions of the Rio de La Plata Grasslands, native grasslands have been substituted by the expansion of short-grass matrix (grassland more adapted to grazing cattle): over the whole study period, the native grassland decreased by $2762.1 \mathrm{~km}^{2}$ in a study area of $21911.4 \mathrm{~km}^{2}$. The fragmentation pattern in the landscape presented spatial differences between analyzed agro-ecological zones, with better conservation status of native grasslands in the Flooding Pampa. The identification of this spatial pattern should promote policies of biodiversity conservation and sustainable management of this landscape.
\end{abstract}

Key words: Grassland, Remote Sensing, GIS, Fragmentation, Pattern.

\section{Resumo}

O presente trabalho, empregou uma combinação de mapas dinâmicos de coberturas e métricas de paisagens baseados em imagens de satélite para quantificar as mudanças espaço-temporais de coberturas e os padrões de fragmentação no centro da província de Buenos Aires (Argentina), no período 1974-2011. Esta área de estudo cobre duas zonas agroecológicas: o Pampa Deprimida e o Pampa Interior. A dife-

* Laboratorio de Investigación y Servicios en Teledetección de Azul-Facultad de AgronomíaUniversidad Nacional del Centro de la Provincia de Buenos Aires (LISTA-FAA-UNCPBA), Av. República de Italia 780, C.C. 47 (7300). Consejo Nacional de Investigaciones Científicas y Técnicas (CONICET) de la Provincia de Buenos Aires, Argentina. 
rença de outras regiões das pastagens do Río de La Plata, a pastagem nativa tem sido substituída por uma matriz de pastos menores (mais adaptada ao pastoreio bovino): durante todo o período de estudo, a pastagem nativa decresceu $2.762,1 \mathrm{~km}^{2}$ em uma área de estudo de $21.911,4 \mathrm{~km}^{2}$. O padrão de fragmentação na paisagem apresentou diferenças espaciais significativas entre as zonas agroecológicas analizadas, com um melhor estado de conservação das pastagens no Pampa Deprimida. A identificação deste padrão espacial deveria promover políticas de conservação da biodiversidade e manejo sustentável desta paisagem.

Palavras chave: Pastiçal, Sensoriamento Remoto, SIG, Fragmentação, Padrão.

\section{Resumen}

En el presente trabajo, se empleó una combinación de mapas dinámicos de coberturas y métricas de paisaje basados en imágenes satelitales para cuantificar los cambios espacio-temporales de coberturas y los patrones de fragmentación en el centro de la provincia de Buenos Aires (Argentina), en el período 1974-2011. Esta área de estudio cubre dos zonas agroecológicas: la Pampa Deprimida y la Pampa Interior. A diferencia de otras regiones de Los Pastizales del Río de La Plata, el pastizal nativo ha sido reemplazado por una matriz de pastos cortos (más adaptada al pastoreo vacuno): durante todo el período de estudio, el pastizal nativo decreció $2762,1 \mathrm{~km}^{2}$ en un área de estudio de $21,911.4 \mathrm{~km}^{2}$. El patrón de fragmentación en el paisaje presentó diferencias espaciales significativas entre las zonas agroecológicas analizadas, con un mejor estado de conservación de los pastizales en la Pampa Deprimida. La identificación de este patrón espacial debería promover políticas de conservación de la biodiversidad y manejo sustentable de este paisaje.

Palabras clave: pastizal, teledetección, SIG, fragmentación, patrón.

\section{Introducción}

Los pastizales son uno de los biomas más extensos del planeta (Sala y Paruelo, 1997). En general, estos sistemas están limitados por la disponibilidad de agua en ciertas épocas del año y poseen un estrato herbáceo dominado por especies de la familia Poaceae. Un pastizal típico del centro de la provincia de Buenos Aires (Argentina) es el pajonal de paja colorada (Paspalum quadrifarium) cuya distribución está caracterizada por presentarse en un amplio rango de topografías (Lara y Gandini, 2013), en donde forma diferentes unidades de vegetación (Perelman et al., 2003, 2005). Durante los últimos cinco siglos, este pastizal ha sido utilizado como recurso forrajero para el ganado doméstico.

Debido a la baja receptividad ganadera del pajonal maduro, el manejo actual se basa en quemas periódicas que induce la mejora a corto plazo en la productividad neta, calidad y accesibilidad del rebrote (Laterra et al., 2003). La interacción del 
fuego con el pastoreo vacuno provoca cambios profundos que pueden percibirse a diferentes escalas de análisis (Herrera et al., 2009; Lara y Gandini, 2011). Este manejo induce la pérdida y fragmentación del pajonal, un proceso que involucra cambios en la composición, configuración y función del paisaje (McGarigal et al., 2005). Además, la fragmentación cambia la composición florística de la comunidad promoviendo la introducción de especies exóticas, muchas de ellas no palatables (Laterra y Maceira, 1996; Paruelo et al., 2004; Lara y Gandini, 2011), y afecta numerosos servicios ecosistémicos como el secuestro de carbono, recursos genéticos, conservación de los suelos, regulación hidrológica y mantención de hábitat para diversas especies de aves y mamíferos (Ameghino, 1884; Costanza et al., 1997; Sala y Paruelo, 1997; Bilenca y Miñarro, 2004).

Estudios similares se han llevado a cabo sobre otras regiones de los Pastizales del Río de La Plata (Soriano et al., 1991; Demaría et al., 2008), donde los pastizales nativos han sido sustituidos por cultivos anuales. Sin embargo, en el centro de la provincia de Buenos Aires (Argentina), núcleo de las actividades productivas de la región, aún no ha sido evaluado el reemplazo del pajonal a escala de paisaje.

El contexto político y socio-económico mundial de los últimos 40 años, principalmente en términos del precio relativo de los granos sobre los productos ganaderos, sometió a la Pampa Argentina a un profundo cambio en la estructura agraria, provocando una reducción del área destinada a la actividad ganadera (Barsky, 1993; Tsakoumagkos, 2003). Según Bilello (2005) el proceso de transformación productiva de la Pampa Argentina, conocido como "agriculturización", comenzó en la década de los setenta con la modernización en el cultivo del maíz y continuó en los ochenta y noventa con la adición de soja en suelos de áreas mixtas históricamente ocupadas por el ganado. Este proceso causa una concentración de la actividad ganadera en suelos de bajo potencial agrícola, provocando una intensificación de uso lo que conlleva al reemplazo o la modificación de los pastizales naturales. Este cambio en tipos de coberturas y área bajo diferentes usos puede ser monitoreada utilizando técnicas de sensoramiento remoto y análisis en sistemas de información geográfica (Oetter et al., 2000; Shi et al., 2012; Tovar et al., 2012).

La información sobre el crecimiento de las prácticas productivas que involucran el reemplazo de pastizales nativos se requiere para poder determinar la magnitud y la tasa a la que ha cambiado el paisaje en las décadas recientes. Este conocimiento es clave para estimar la escala espacio-temporal a la cual los procesos operan y se convierte en esencial para la implementación de estrategias de conservación y manejo sustentable de los pastizales de la región.

La teledetección es una poderosa herramienta para evaluar la dinámica espaciotemporal del uso y cobertura de la tierra a lo largo del paisaje (Shi et al., 2012). Además, las métricas de paisaje son importantes porque proveen una medición total de los procesos ecológicos y los efectos del cambio de uso y cobertura de la tierra 
sobre la fragmentación del paisaje (Wu y Hobbs, 2002; Southworth et al., 2004; Cushman et al., 2008; Herrera et al., 2009; Shi et al., 2012). Los objetivos del presente trabajo fueron identificar los cambios de cobertura en el período 1974-2011 y evaluar el estado de fragmentación del pajonal en el centro de la provincia de Buenos Aires, Argentina.

\section{Características del área de estudio}

Este trabajo se llevó a cabo en la región central de la distribución original del pajonal en la provincia de Buenos Aires, Argentina (Vervoorst, 1967), que cubre dos zonas agro-ecológicas: la Pampa Deprimida y la Pampa Interior (Gandini et al., 2014) (Figura 1). El área de estudio es una depresión muy ancha de $21,911.39 \mathrm{~km}^{2}$, con una escasa pendiente que oscila entre $0.025 \%$ y $0.5 \%$. Finos sedimentos de origen eólico (loess) del Pleistoceno y Holoceno cubren esta región, con suelos hidro-halomórficos que son afectados por inundaciones. Pastizales naturales o seminaturales dedicados al pastoreo vacuno cubrían la mayoría de la región (Tricart, 1973).

El clima es templado-húmedo con una precipitación media anual de $970 \mathrm{~mm}$. La temperatura media anual es $13.9^{\circ} \mathrm{C}$; el mes más frío es julio con una temperatura mínima media de $3.3{ }^{\circ} \mathrm{C}$ y el mes más cálido es enero con una máxima media de $21.2^{\circ} \mathrm{C}$ (Köppen, 1918).

\section{Materiales y métodos}

\section{Adquisición de los datos, pre-procesamiento y clasificación de tipos de cobertura}

Se utilizó una serie temporal de cuatro imágenes Landsat (path 225, row 85) para los años 1974 (sensor MSS), 1988 (sensor TM), 2000 (sensor ETM+) y 2011 (sensor TM). Se hizo especial hincapié sobre una escala temporal óptima de 11-14 años porque los cambios que ocurren en el pajonal son relativamente lentos (Herrera, et al., 2005).

Los números digitales (ND) se convirtieron a valores de reflectancia (excepto la banda térmica) de acuerdo a los coeficientes radiométricos propuestos por Chander et al. (2009), posteriormente los valores de reflectancia fueron corregidos de los efectos atmosféricos utilizando el Método Mejorado de Sustracción de Objetos Oscuros (Chavez, 1996).

Siguiendo trabajos precedentes (Herrera et al., 2005, 2009; Lara y Gandini, 2013), los tipos de coberturas utilizadas fueron las siguientes: pajonal (PJ), matriz de pastos cortos (MPC), pasturas (P), cultivos (C) y cuerpos de agua (CA). Para identificar los diferentes tipos de cobertura se utilizaron clasificaciones supervisadas empleando el algoritmo de máxima verosimilitud. Para las clasificaciones se 


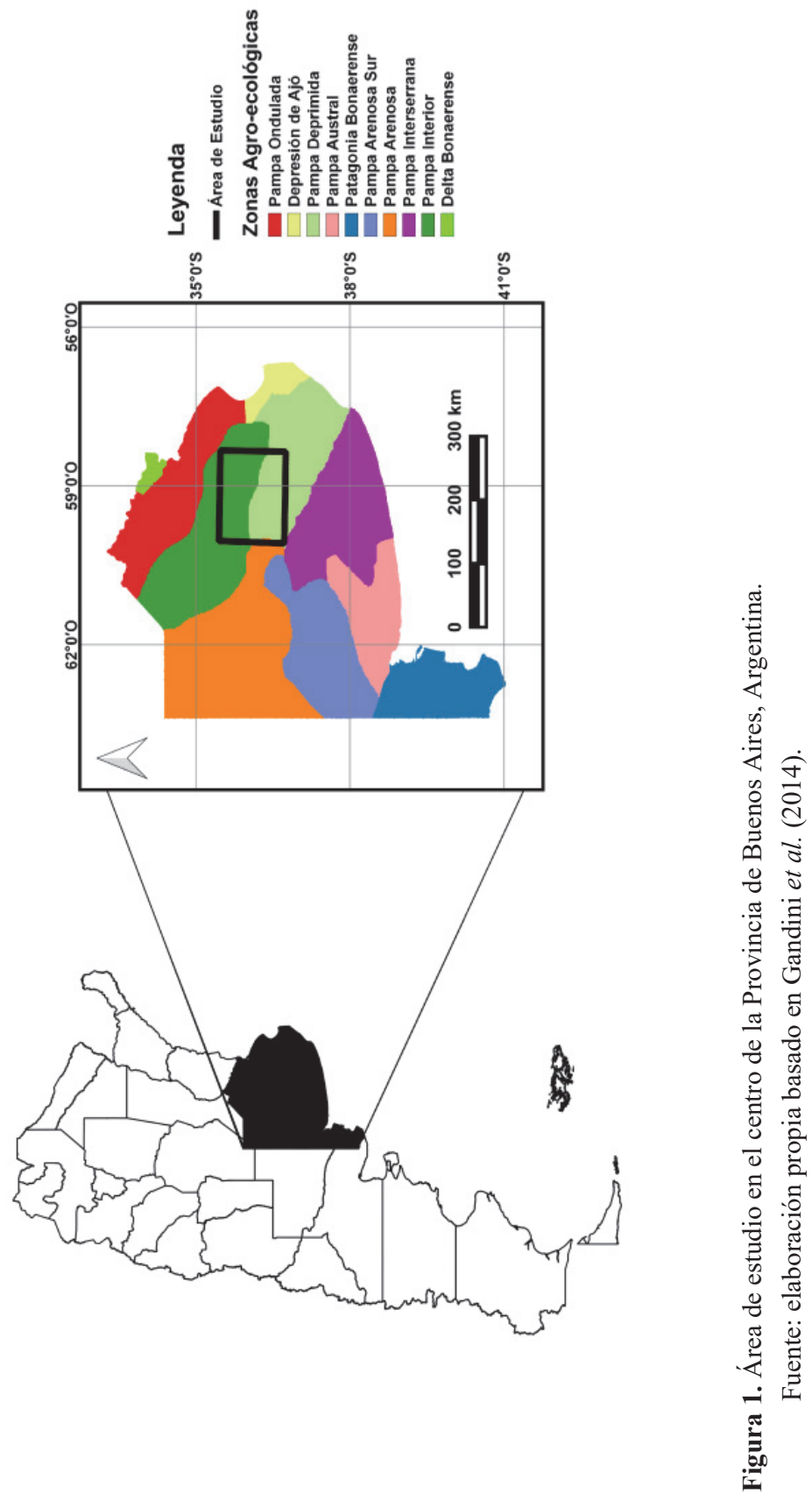



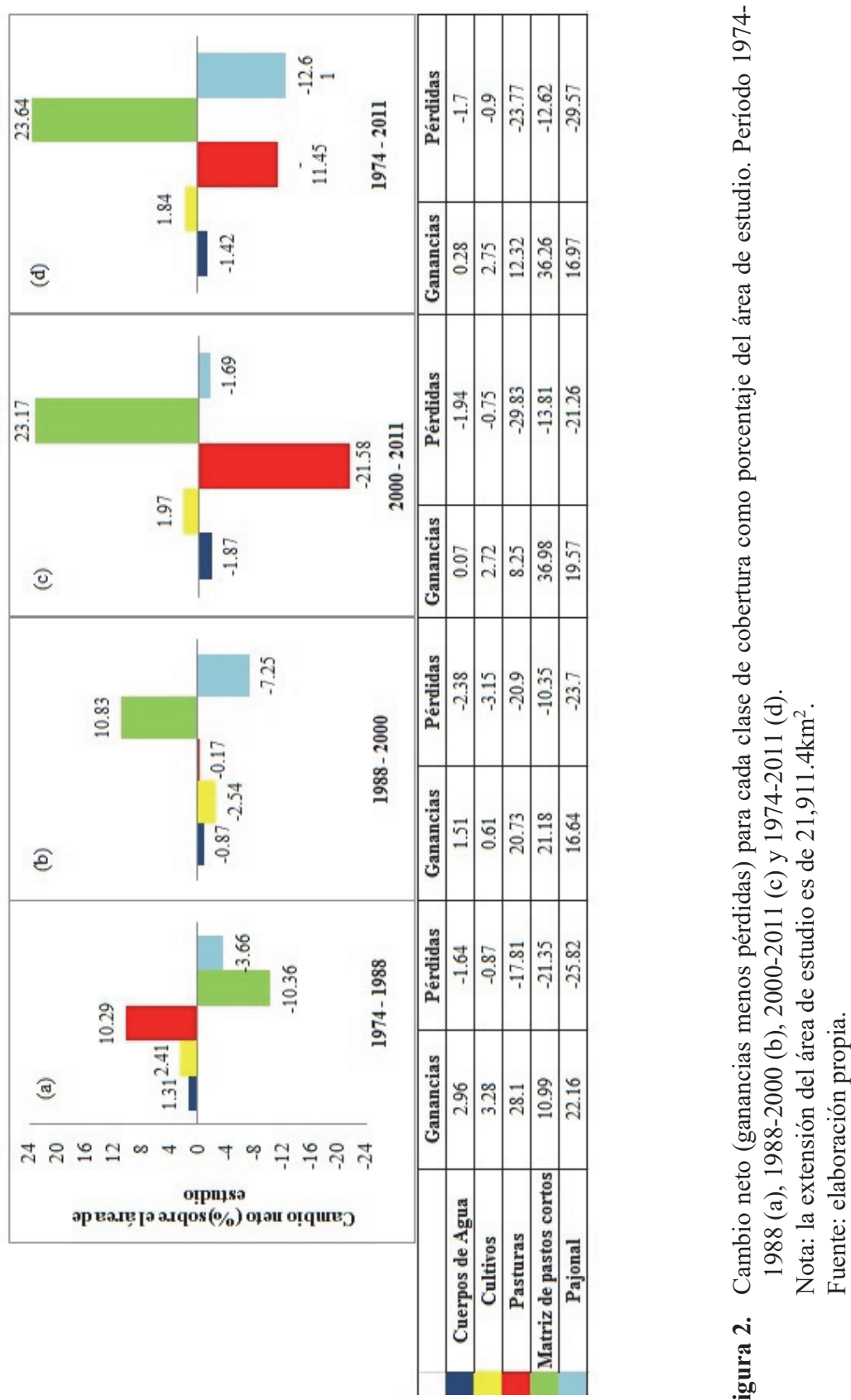

ֻับำ 
utilizaron todas las bandas reflectivas de los satélites Landsat; para el año 1974:MSS4, MSS5, MSS6 y MSS7; para los años 1988 y 2011: TM1, TM2, TM3, TM4, TM5 y TM7; para el año 2000: $\mathrm{ETM}^{+} 1, \mathrm{ETM}^{+} 2, \mathrm{ETM}^{+} 3, \mathrm{ETM}^{+} 4, \mathrm{ETM}^{+} 5$ y $\mathrm{ETM}^{+}$7. Se descartó la banda térmica de los sensores TM y ETM ${ }^{+}$.

Para el año 1988, los sitios de entrenamiento fueron localizados mediante interpretación visual sobre 44 fotografías aéreas infrarrojas (escala 1:20.000), siguiendo criterios tales como textura, forma y color (Chuvieco, 2010). Para el año 2011, los puntos de control fueron seleccionados a campo utilizando un sistema de geoposicionamiento global (GPS) dentro de áreas relativamente homogéneas. Tanto para el año 1974 y 2000, los sitios de entrenamiento fueron seleccionados por análisis visual teniendo en cuenta la firma espectral promedio para cada uno de los tipos de coberturas de interés y utilizando áreas con características espectrales similares (Chuvieco, 2010; Schulz et al., 2010).

Los resultados de la clasificación fueron filtrados utilizando un filtro de mediana de 7 x 7 para remover los pixeles aislados (el efecto "sal y pimienta"). Posteriormente, la clasificación de la imagen de 1974 (sensor MSS) fue re-muestreada a un tamaño de pixel de $30 \mathrm{~m}$ x $30 \mathrm{~m}$ para permitir la comparación multitemporal con el resto de la serie. Una vez obtenidos los mapas de coberturas, se evaluaron las precisiones de las clasificaciones mediante el estadístico Kappa (Congalton, 1991). Este estadístico, basado en una matriz de confusión, tiene en cuenta los errores de comisión (o inclusión) y los errores de omisión (o exclusión), siendo más precisa la clasificación cuanto más cercano a un valor de 1 se encuentre, cuantificando la performance de una clasificación particular con respecto a una asignación al azar de pixeles a cada clase.

\section{Análisis de los cambios de coberturas}

La determinación de los cambios de coberturas fue realizada con el Modelador de Cambios de Uso del Suelo de Idrisi (Eastman, 2009), el cual es una aplicación vertical que examina y estima los cambios (y transiciones) a través de la matriz de tabulación cruzada (Pontius et al., 2004). De esta manera, el mapeo de la distribución espacial de las transiciones, persistencias, ganancias y pérdidas se obtuvieron con el Modelador de Cambios de Uso del Suelo. Los mapas binarios de cambio/no cambio se realizaron para cada período para crear un mapa de persistencias y cambios para el área de estudio. Los tres mapas resultantes fueron sumados para obtener un mapa de persistencia e intensidad de cambios para el período global. Los pixeles persistentes representan aquellos que nunca cambiaron de tipo de cobertura, y la intensidad de los cambios está dada por los pixeles que muestran 1, 2 ó 3 cambios a través de los distintos períodos estudiados (1974-1988, 1988-2000 y 2000-2011). Por lo tanto, un valor de 3 está asociado con el mayor grado de intensidad y un valor de 1 con el menor grado de intensidad de cambio. 


\section{Análisis de la fragmentación del paisaje}

Los patrones del paisaje se cuantificaron para las dos zonas agroecológicas del área de estudio (la Pampa Deprimida y la Pampa Interior). Dentro de cada sector se delimitaron 10 ventanas de análisis de 12 x 12km (14,400ha, cada una considerada como un paisaje) y se analizaron con FragStats 4.1 (McGarigal y Ene, 2012). Las métricas de paisaje han sido criticadas por muchos investigadores (Wu y Hobbs, 2002; Li y Wu, 2004; Frohn y Hao, 2006). Sin embargo, no hay una manera objetiva para descartar algunas métricas o una "receta" para agrupar las más importantes (Matteucci y Silva, 2005). En este sentido, utilizamos aquellas métricas que representen la mayor variabilidad en el paisaje y nos permita conocer rápida y fácilmente la configuración estructural de nuestra área de estudio. Además, la mayoría de ellos han sido útiles para cuantificar los aspectos principales de la configuración espacial de diferentes paisajes (McGarigal y Marks, 1995; Heilman et al., 2002; Matteucci y Silva, 2005; McGarigal et al., 2005; Cushman et al., 2008).

Por lo tanto, en este trabajo, se emplearon ocho métricas las cuales fueron obtenidas sólo para la clase "pajonal". Estas métricas son: 1) porcentaje del paisaje (PLAND); 2) densidad de parches (PD); 3) índice de parche mayor (LPI); 4) densidad de borde (ED); 5) índice de forma (LSI); 6) área media de la clase (CA); 7) distancia euclidea al vecino más cercano (ENN); y 8) índice de dispersión y yuxtaposición (IJI). A escala de paisaje, los patrones de fragmentación del pajonal fueron evaluados entre zonas agroecológicas mediante el test de t para la comparación de medias. Este procedimiento se realizó tanto para el mapa inicial (1974) como para el mapa final (2011).

\section{Resultados y discusión}

\section{Evaluación de la precisión}

Se obtuvieron muy buenas precisiones en las clasificaciones para los años 1974, 2000 y 2011 con un índice Kappa de $0.800,0.887$ y 0.885, respectivamente; y una buena precisión para la clasificación de 1988, con un Kappa de 0.653. Además, la clase pajonal estuvo bien discriminada del resto de las coberturas del suelo con valores de precisiones del productor y el usuario superiores a 69\% y 63\%, respectivamente. En general, la principal confusión se da entre el pajonal y la matriz de pastos cortos, debido a su similar comportamiento espectral independientemente de la estación de crecimiento (Lara y Gandini, 2013). La distribución espacial de los pastizales es compleja y raramente homogénea (Vervoorst, 1997). Además, el área de estudio es un ambiente donde los pixeles puros son raros lo que contribuye a la heterogeneidad espectral del paisaje. Éstos son problemas inherentes en la clasifica- 

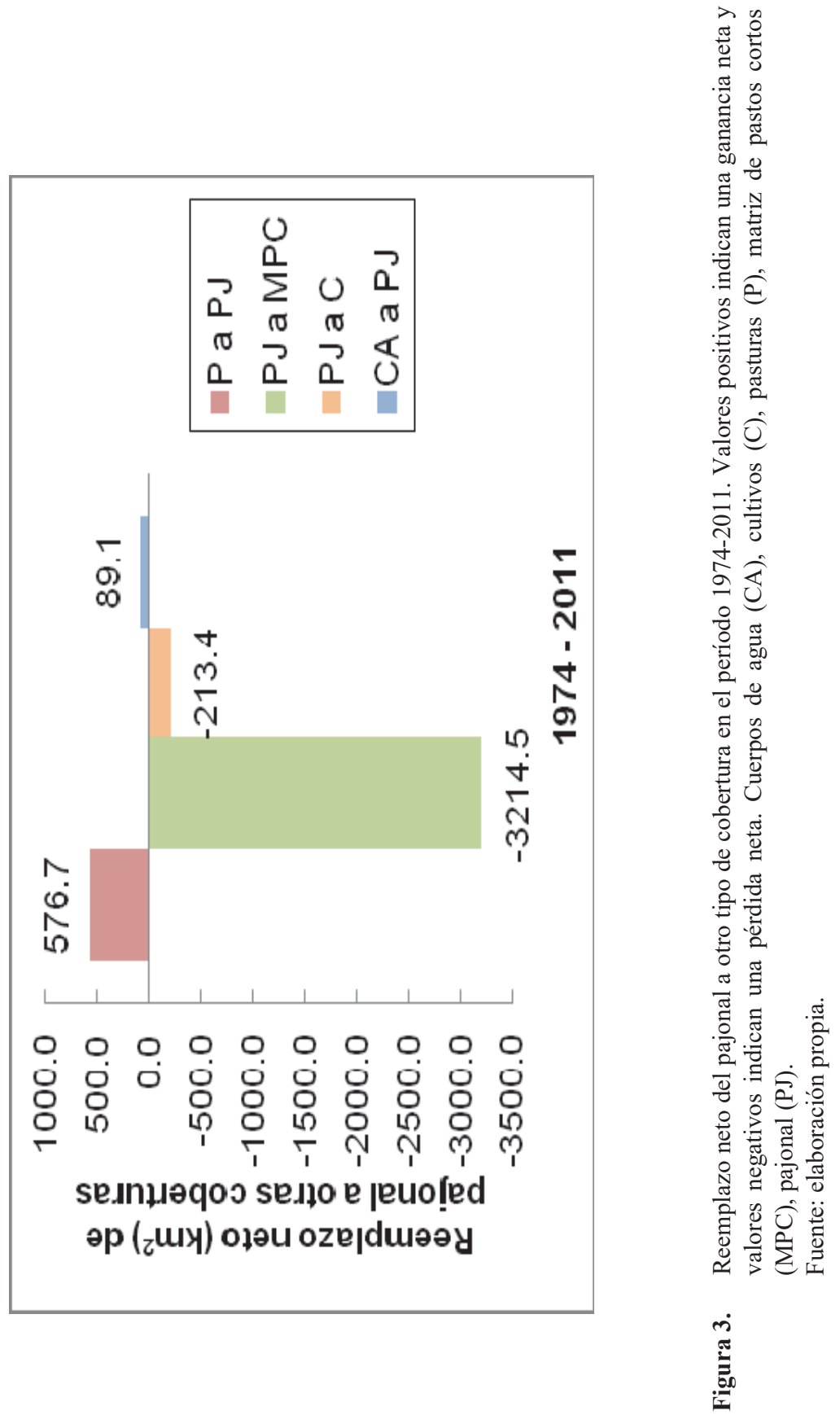


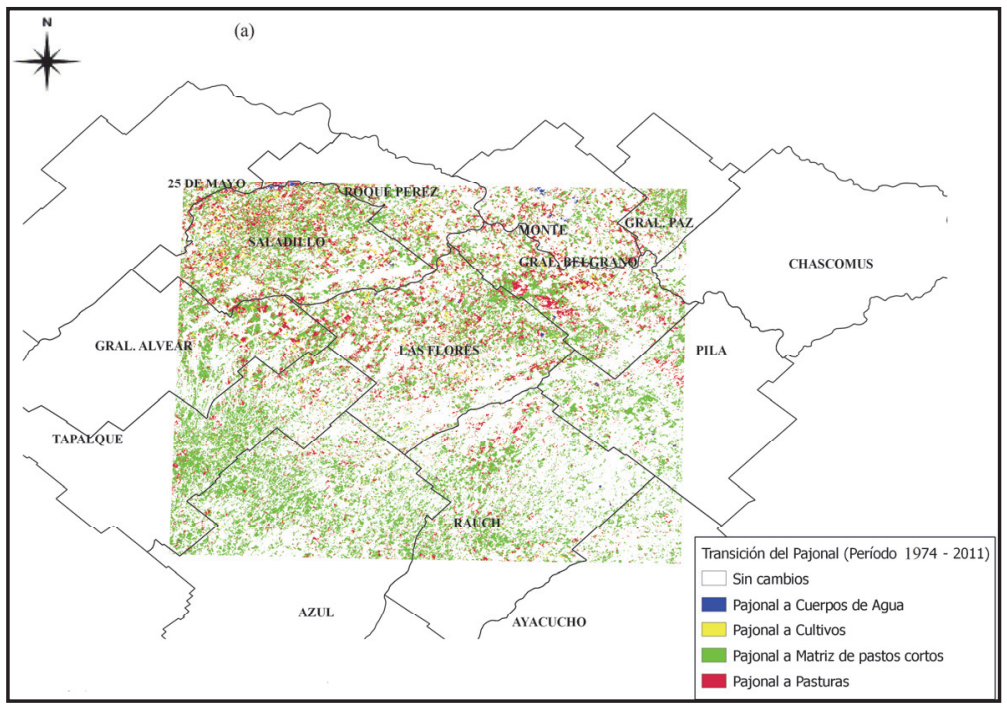

a

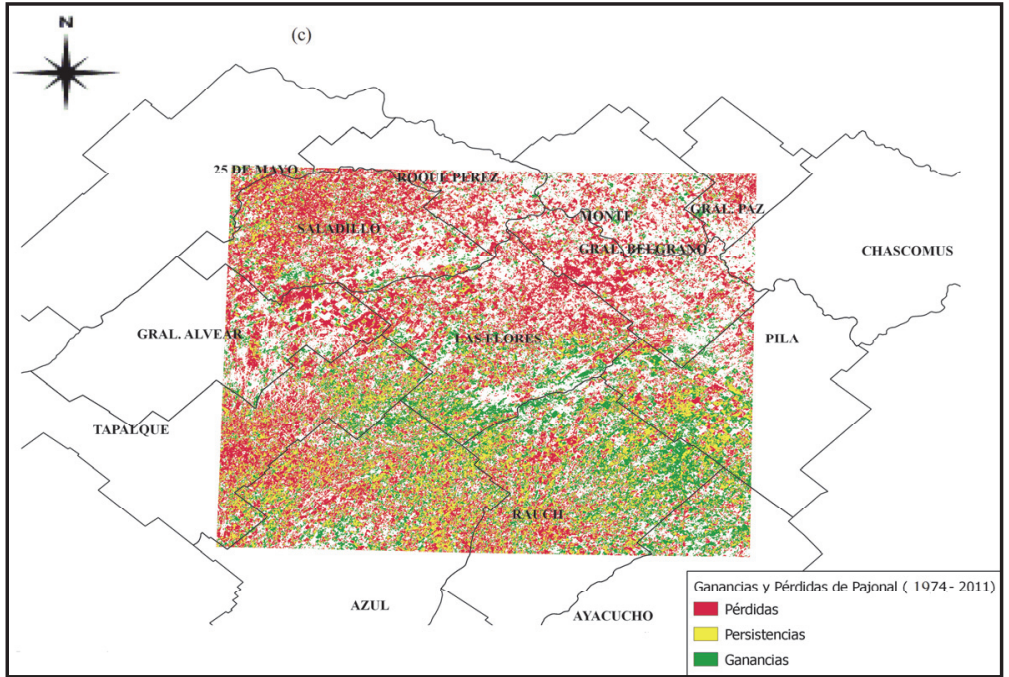




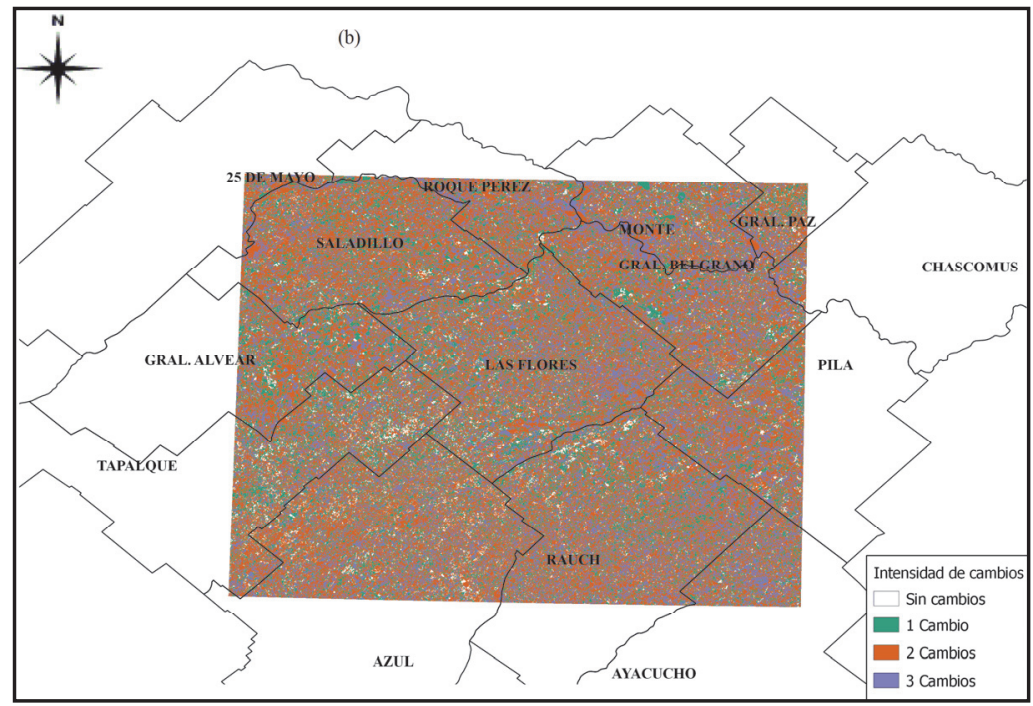

c

Figura 4. (a) Distribución espacial de la transición del pajonal a otras coberturas en el período 1974-2011. (b) Distribución espacial de la intensidad de cambios y los patrones de cambio de cobertura y persistencia a lo largo de los tres períodos. (c) Patrones de colonización y pérdida de parches de pajonal, incluyendo los parches pre-existentes.

Fuente: elaboración propia.

ción de pastizales, especialmente en imágenes de media resolución como las utilizadas en este trabajo. Sin embargo, la clasificación empleada permitió obtener muy buenas precisiones del usuario y del productor para la clase pajonal, similar a la encontrada por otros investigadores (Oetter et al., 2000; Price et al., 2002; Herrera et al., 2005; Demaría et al., 2008; Argañaraz y Entraigas, 2008; Shi et al., 2012; Tovar et al., 2012).

\section{Cambios de coberturas y distribución espacial de los cambios}

Durante el período global (1974-2011), el pajonal disminuyó 2,762.1 km². Este tipo de cobertura ocupó 43.2\% y 30.6\% del área de estudio en 1974 y 2011, respectivamente. Esta pérdida no fue constante: para el período $1974-1988,801.9 \mathrm{~km}^{2}$ fueron convertidos a otro tipo de coberturas; para 1988-2000, este valor incrementó a $1,5896 \mathrm{~km}^{2}$, mientras que para el período 2000-2011, el pajonal disminuyó $370.7 \mathrm{~km}^{2}$.

Para el período 1974-2011 se observó un gran incremento de 5,179.9km² (23.6\% del área de estudio) de la matriz de pastos cortos. También se encontró un 
incremento en la superficie de cultivos anuales $\left(403.9 \mathrm{~km}^{2} ; 1.8 \%\right.$ del área de estudio), al mismo tiempo que se observó una disminución en la superficie cubierta por las pasturas $\left(2,509.5 \mathrm{~km}^{2} ; 11.5 \%\right.$ del área de estudio) (Figura 2). Por otro lado, el pajonal fue principalmente sustituido por la matriz de pastos cortos $\left(3,214.5 \mathrm{~km}^{2}\right) \mathrm{y}$, en menor proporción, por los cultivos $\left(213.4 \mathrm{~km}^{2}\right)$. Además, se encontró una transición de pastura a pajonal de $576.7 \mathrm{~km}^{2}$ (Figura 3). A diferencia de otras regiones de los Pastizales del Río de La Plata donde los pastizales nativos fueron reemplazados por cultivos (Soriano et al., 1991; Demaría et al., 2008), el reemplazo del pajonal en este trabajo es mejor explicado por la expansión de la matriz de pastos cortos (un tipo de pastizal más adaptado al pastoreo vacuno; Figura 4a). Esto se debe a que, en esta región, los suelos presentan una baja capacidad agrícola, con severas limitaciones como inundaciones periódicas, alta salinidad y un estrato calcáreo.

La distribución espacial de la intensidad y los patrones de los cambios de cobertura y las persistencias se muestran en la Figura $4 \mathrm{~b}$ y $4 \mathrm{c} ; 23.4 \%$ del área de estudio estuvo sujeta a solamente un cambio, 45.2\% a dos cambios, 27.3\% mostró cambios en los tres períodos analizados y solamente el $4.3 \%$ de los pixeles permanecieron sin cambios. La mayoría de los pixeles sin cambios pertenecen a la clase pajonal $(55.1 \%)$ y pasturas $(26.4 \%)$, seguidos por la matriz de pastos cortos $(18.5 \%)$, cuerpos de agua $(0.02 \%)$ y cultivos $(0.005 \%)$.

La intensidad de los cambios de cobertura no mostró un patrón espacial definido, sino que los cambios se distribuyen aleatoriamente en toda el área de estudio. Esta ausencia de un patrón espacial puede estar asociada con las estrategias de manejo basadas en quemas periódicas y ciclos de cultivos/pasturas que permitieron el establecimiento de estados alternativos de pastizales (Laterra et al., 1998, 2003). Este resultado es diferente al producido por la intensificación de la agricultura, la cual avanza sobre los mejores suelos y muestra un patrón espacial característico (Schulz et al., 2010). La distribución espacial de los cambios de coberturas observados en este trabajo está relacionada a factores físicos y socio-económicos. El contexto mundial ha estimulado el avance de la agricultura sobre áreas históricamente ocupadas por la ganadería, concentrando el pastoreo sobre suelos de muy baja aptitud agrícola (Barsky, 1993; Tsakoumagkos, 2003; Bilello, 2005). Este proceso ha dado lugar a una intensificación sin precedentes, que conduce a la pérdida y fragmentación de los pastizales naturales.

En la actualidad, se está llevando a cabo el proyecto "Pastizales y Sabanas del Cono Sur de Sudamérica: Iniciativas para su conservación en Argentina" coejecutado por Aves Argentinas-AOP (AA) y Fundación Vida Silvestre Argentina (FVSA) con financiamiento del Fondo de Medio Ambiente Mundial (GEF, por sus siglas en inglés) a través del Banco Internacional para la Reconstrucción y Fomento (Banco Mundial). El objetivo de este proyecto es impulsar la ganadería sustentable en pastizales promoviendo la integración de la conservación de la naturaleza y la 
producción agropecuaria $<$ http://www.alianzadelpastizal.org/en/ $>$. De este modo, se podría lograr un manejo que vincule la producción y la conservación de los pastizales naturales.

\section{Recolonización del pajonal}

Un gran número de nuevos parches de pajonal se encontraron donde antes había otro tipo de coberturas (Figura 4c). Estos parches estaban asociados con grandes parches pre-existentes de pajonal. Este hecho apoya la idea de que la conservación de grandes remanentes de pajonal es esencial para la restauración del paisaje, ya que Paspalum quadrifarium posee una pobre capacidad de dispersión por semilla (Laterra et al., 1998). Además, este proceso, siempre que vaya acompañado por un régimen de gestión integral, ayudaría a garantizar la estabilidad de los diferentes servicios ecosistémicos a escala de paisaje (Costanza et al., 1997; Bilenca y Miñarro, 2004).

Esta re-colonización del pajonal ha sido observada en un modelo de estados y transiciones basado en observaciones de corto tiempo. Según Laterra et al. (1998), Paspalum quadrifarium puede establecerse con éxito a partir del banco de semillas en sitios perturbados por el fuego y con baja carga ganadera. La planificación del paisaje y la conservación de los ecosistemas en esta área son en la actualidad de gran importancia en la Argentina, debido a la creciente presión sobre los recursos naturales por la intensificación agrícola y la producción ganadera.

\section{Análisis de la fragmentación del paisaje}

No se encontraron diferencias significativas en los patrones espaciales de fragmentación del pajonal entre las zonas agroecológicas para el mapa de 1974 (Tabla 1). Sin embargo, el pajonal en la Pampa Deprimida presentó valores más altos que en la Pampa Interior en el porcentaje del paisaje (PLAND) y el índice de parche mayor (LPI), las cuales son métricas con un valor considerable en conservación (McGarigal et al., 2005). Para el mapa de 2011, se encontraron diferencias significativas en los patrones espaciales de fragmentación del pajonal entre ambas zonas agroecológicas (Tabla 1). El porcentaje ocupado por el pajonal (PLAND) fue más alto en la Pampa Deprimida que en la Pampa Interior (42.0\% y 17.1\%, respectivamente). El índice de parche mayor (LPI) difirió entre zonas agroecológicas, siendo más alto en la Pampa Deprimida. Además, la distancia euclidea al vecino más cercano (ENN) entre parches de pajonal fue más alta en la Pampa Interior que en la Pampa Deprimida. Sin embargo, no se encontraron diferencias significativas entre las zonas agroecológicas para el área promedio total de la clase (CA) y el índice de forma del paisaje (LSI). El índice de dispersión y yuxtaposición (IJI) presentó diferencias 
Tabla 1

Patrones espaciales de fragmentación del pajonal entre zonas agroecológicas para los mapas de 1974 y 2011. p-valor $<0.05$ indican diferencias significativas

\begin{tabular}{|c|c|c|c|c|c|c|}
\hline & \multicolumn{3}{|c|}{ Mapa 1974} & \multicolumn{3}{|c|}{ Mapa 2011} \\
\hline $\begin{array}{c}\text { Métricas } \\
\text { de paisaje }\end{array}$ & $\begin{array}{c}\text { Pampa } \\
\text { Deprimida }\end{array}$ & $\begin{array}{l}\text { Pampa } \\
\text { Interior }\end{array}$ & p-valor & $\begin{array}{c}\text { Pampa } \\
\text { Deprimida }\end{array}$ & $\begin{array}{l}\text { Pampa } \\
\text { Interior }\end{array}$ & p-valor \\
\hline PLAND (\%) & 57.5 & 50.0 & 0.20 & 42.0 & 17.1 & 0.00005 \\
\hline PD (parches. $100 \mathrm{ha}^{-1}$ ) & 0.5 & 0.6 & 0.64 & 0.9 & 1.2 & 0.07 \\
\hline LPI $(\%)$ & 48.3 & 35.4 & 0.16 & 22.8 & 3.7 & 0.009 \\
\hline $\operatorname{ED}\left(m \cdot h a^{-1}\right)$ & 32.7 & 31.2 & 0.30 & 31.2 & 20.6 & 0.0003 \\
\hline LSI & 13.8 & 14.1 & 0.74 & 15.5 & 15.5 & 0.98 \\
\hline CA (ha) & 169.9 & 191.2 & 0.86 & 68.0 & 14.1 & 0.06 \\
\hline ENN (m) & 149.5 & 149.3 & 0.99 & 153.9 & 215.3 & 0.0009 \\
\hline IJI (\%) & 39.6 & 32.8 & 0.18 & 26.7 & 39.4 & 0.009 \\
\hline
\end{tabular}

significativas entre zonas, pero ambos valores fueron bajos (Tabla 1), lo que indica que la clase pajonal se encuentra entremezclado con pocos tipos de coberturas (posiblemente la matriz de pastos cortos y las pasturas).

La variación temporal de las principales métricas presenta la misma tendencia, indicando una pérdida sostenida de grandes parches de pajonal. Sin embargo, el patrón de fragmentación fue significativamente diferente entre zonas agroecológicas, siendo de mayor intensidad en la Pampa Interior. Esta zona mostró una disminución en el porcentaje ocupado por el pajonal y en el índice de parche mayor, acompañado por un aumento en el aislamiento entre parches de pajonal. Estos resultados son comparables a las descripciones realizadas por varios investigadores (Forman, 1995; McGarigal y Marks, 1955; Li y Wu, 2004; McGarigal et al., 2005) quienes indicaron patrones similares en diferentes paisajes fragmentados.

En la Pampa Deprimida, el pajonal presenta un menor grado de fragmentación, lo que indica un mejor estado de conservación posiblemente debido a una menor intensificación de las actividades productivas, presumiblemente, por las condiciones adversas de sus suelos. En el área de estudio, prevalecen los establecimientos privados y el constante reemplazo del pajonal es el principal problema para la conservación de este pastizal nativo, ya que esta comunidad no está representada dentro de áreas protegidas ni públicas ni privadas (Bilenca y Miñarro, 2004; Lara y Gandini, 2013). Los parches de pajonal encontrados en este trabajo representan una estructura similar al pastizal original que dominó en el paisaje de la Pampa Argentina en el 
pasado y juega un importante rol en la mantención de la biodiversidad, de los servicios ecosistémicos y en los patrones estructurales de la diversidad de especies (Costanza et al., 1997; Laterra et al., 2003; Perelman et al., 2003; Bilenca y Miñarro, 2004).

A pesar de los esfuerzos por regular la carga ganadera y mejorar la disponibilidad de forraje (Vecchio et al., 2008; Rodríguez y Jacobo, 2012), la implementación de planes de manejo integrales aún continúa siendo uno de los principales desafíos para la conservación de pastizales naturales a escala de paisaje.

\section{Conclusiones}

Basados en los mapas de coberturas, derivados de datos de sensores remotos y con la ayuda de herramientas de los sistemas de información geográfica se ha cuantificado y evaluado la distribución del patrón espacial del paisaje y sus cambios en el centro de la provincia de Buenos Aires (Argentina). En contraste con lo que ocurre en otras regiones del país, donde los pastizales nativos han sido sustituidos por cultivos agrícolas, el reemplazo del pajonal (la comunidad nativa de mayor relevancia de la región) es mejor explicado por la expansión de un tipo de pastizal más adaptado al pastoreo vacuno, es decir, la matriz de pastos cortos. A lo largo del total del período analizado, el pastizal nativo disminuyó $2,762.1 \mathrm{~km}^{2}$ en un área de estudio de $21,911.4 \mathrm{~km}^{2}$, y la matriz de pastos cortos se incrementó en $5,179.9 \mathrm{~km}^{2}$. En cambio, la superficie cubierta por cultivos anuales solamente se incrementó en $403.9 \mathrm{~km}^{2}$.

A pesar de que el pajonal en la Pampa Interior presenta un alto grado de fragmentación, en la Pampa Deprimida aún permanece en un buen estado relativo de conservación. El pajonal, en la Pampa Interior, mostró una marcada disminución en el porcentaje del paisaje (de $50.0 \%$ a $17.1 \%$ ) y en el índice de parche mayor (de $35.4 \%$ a 3.7\%). En cambio, en la Pampa Deprimida, mostró una disminución más suave en esas métricas: de $57.5 \%$ a $42.0 \%$ y de $48.3 \%$ a $22.8 \%$, respectivamente. Estos resultados sugieren que el proceso de fragmentación fue más severo en la Pampa Interior, indicando un mejor estado de conservación en la Pampa Deprimida. Además, las métricas recientemente mencionadas son algunas de las más importantes a la hora de mantener estables los servicios ecosistémicos y la biodiversidad vegetal y animal.

Por otro lado, la conservación de grandes parches remanentes de pajonal ha mostrado ser muy importante para la re-colonización debido a su reducida capacidad de dispersión por semilla. La identificación de este patrón espacial de fragmentación y re-colonización del pajonal debería ser esencial y motivar la promoción de políticas de conservación de la biodiversidad y manejo sustentable de este paisaje.

Las principales contribuciones de este trabajo fueron cuantificar, localizar y mapear los cambios de coberturas en la Pampa Argentina, núcleo productivo del 
país, a través de 37 años. Además, el análisis de los patrones de fragmentación nos permitió caracterizar la intensidad de uso del pastizal natural por el pastoreo vacuno y sus prácticas asociadas. De esta manera, los parches de pajonal mejor preservados deberían ser tomados en cuenta para la implementación de áreas protegidas de pastizales nativos.

\section{Bibliografía}

Ameghino, F. (1884). Las secas y las inundaciones en la provincia de Buenos Aires, 5a. edición, Ministerio de Asuntos Agrarios de la Provincia de Buenos Aires, $62 \mathrm{pp}$.

Argañaraz, J.P. y Entraigas, I. (2011). “Análisis comparativo entre las máquinas de vectores soporte y el clasificador de máxima probabilidad para la discriminación de cubiertas del suelo", Revista de Teledetección, núm. 36, pp. 26-39.

Barsky, O. (1993). "La evolución de las políticas agrarias en Argentina”, en Bonaudo, M. y Pucciarelli, A. (comps.), La problemática agraria. Nuevas aproximaciones, vols. I, II y III, Buenos Aires, Centro Editor de América Latina, 51-88 pp.

Bilello, G. (2005). "Estrategias productivas y posibilidad de permanencia. La evolución de los pequeños productores de Azul en el marco de la globalización", en González, M., Productores familiares pampeanos: hacia la comprensión de similitudes y diferenciaciones zonales, 1a. ed., Editorial Astralib Cooperativa, Buenos Aires.

Bilenca, D. y Miñarro, F. (2004). Identificación de áreas valiosas de pastizal (AVPs) en las pampas y campos de Argentina, Uruguay y sur de Brasil, Fundación Vida Silvestre, Buenos Aires.

Chander, G. Markham, B. y Helder, D.L. (2009). "Summary of current radiometric calibration coefficients for Landsat MSS, TM, ETM+, and EO-1 ALI sensors", Remote Sensing of Environment, no. 113, pp. 893-903.

Chavez, Jr. P. (1996). "Image-based atmospheric correction- Revisited and improved", Photogrammetric Engineering and Remote Sensing, vol. 2, no. 9, pp. 1025-1036.

Chuvieco, E. (2010). Teledetección ambiental, Editorial Ariel, Madrid.

Congalton, R.G. (1991). "A review of assessing the accuracy of classifications of remotely sensed data", Remote Sensing of Environment, no. 37, pp. 35-46.

Costanza, R., D’Arget, R., Groot, R., Faber, S., Grasso, M., Hannon, B., Limburg, K., Naeem, S., O’Neill, T., Paruelo, J., Sutton, R. y Van den Belt, M. (1997). "The value of the world's ecosystem services and natural capital", Nature, no. 387, pp. 253-260. 
Cushman, S.A., McGarigal, K. y Neel, M. (2008). "Parsimony in landscape metrics: strength, universality and consistency”, Ecological Indicators, no. 8, pp. 691703.

Demaría, R., Aguado, I. y Steinaker, D. (2008). "Reemplazo y fragmentación de pastizales pampeanos semiáridos en San Luis, Argentina", Ecología Austral, núm. 18 , pp. 55-70.

Eastman, J.R. (2009). Idrisi Taiga Tutorial. Land Change Modeler, Clark Labs., Clark University, Wourcester, MA, USA.

Forman, R.T. (1995). Land mosaics: the ecology of landscapes and regions, Cambridge University Press, New York.

Gandini, M.L., Lara, B. y Scaramuzzino, R. (2012). "Zonificación basada en la respuesta de ecosistemas a oscilaciones climáticas", Revista Estudios Ambientales, vol. 2, núm. 2, pp. 4-24.

Heilman, G.E., Strittholt, J., Slosser, N. y Dellasala, N. (2002). "Forest fragmentation of the conterminous United States: Assessing forest intactness through road density and spatial characteristics", BioScience, vol. 52, no. 5, pp. 411-422.

Herrera, L., Gómez Hermida, V., Martínez, G., Laterra, P. y Maceira, N. (2005). "Remote Sensing Assessment of Paspalum quadrifarium Grasslands in the Flooding Pampa, Argentina", Rangeland Ecology and Management, vol. 58, no. 4, pp. 406-412.

Herrera, L., Laterra, P., Maceira, P., Zelaya, K. y Martínez, G. (2009). "Fragmentation status of tall-tussock grassland relicts in the Flooding Pampa, Argentina", Rangeland Ecology and Management, no. 62, pp. 73-82.

Köppen, W. (1918). "Klassifikation der klimate nach temperatur, niederschlag, und jahreslauf”, Petermann's Mitteilungen, no. 64, pp. 193-203.

Lara, B. y Gandini, M. (2011). "Biogeografía de islas en fragmentos de pajonal del Paisaje Ariel (Azul, Buenos Aires, Argentina)", Revista de la Asociación Argentina de Ecología de Paisajes, núm. 2, pp. 1-8.

(2013). "Nuevo aporte para la distribución del Paspaletum en el centro de la provincia de Buenos Aires", Revista de la Asociación Argentina de Ecología de Paisajes, núm. 4, pp. 1-12.

Laterra, P. y Maceira, N. (1996). "Ecología de pajonales de paja colorada: impacto del fuego sobre la productividad, la biodiversidad y la estabilidad del sistema", en Sarmiento, G. y M. Cabido (eds.), Biodiversidad y funcionamiento de Pastizales y Sabanas en América Latina. Estado del conocimiento y perspectivas de investigación, Cyted-Cielat.

Laterra, P., Vignolio, O., Hidalgo, L., Fernández, O., Cahuépé, M. y Maceira, N. (1998). "Dinámica de pajonales de paja colorada (Paspalum spp.) manejados con fuego y pastoreo en la Pampa Deprimida Argentina", Ecotrópicos, vol. 11 núm. 2, pp. 141-149. 
Laterra, P., Vignolio, O., Linares, M., Giaquinta, A. y Maceira, N. (2003). "Cumulative effects of fire on a tussock pampa grassland", Journal of Vegetation Science, no. 14, pp. 43-54.

Li, H. y Wu, J. (2004). "Use and misuse of landscape indices", Landscape Ecology, no. 19 , pp. 389-399.

Matteucci, S.D. y Silva, M. (2005). "Selección de métricas de configuración especial para la regionalización de un territorio antropizado", Geofocus (Artículos) núm. 5, pp. 180-202.

McGarigal, K. y Marks, B. (1995). Fragstats: spatial pattern analysis program for quantifying landscape structure. US Departament of Agriculture, Forest Service, Northwest Research Station, USA, Portland, OR.

McGarigal, K. y Ene, E. (2012). Fragstats 4.1. A spatial pattern analysis program for categorical maps.

McGarigal, K., Cushman, S. y Regan, C. (2005). Quantifying terrestrial habitat loss and fragmentation: A protocol, University of Massachusetts.

Oetter, D., Cohen, W., Berterretche, M., Maiersperger, T. y Kennedy, R. (2000). "Land cover mapping in an agricultural setting using multiseasonal Thematic Mapper data", Remote Sensing of Environment, no. 76, pp. 139-155.

Paruelo, J., Piñeiro, G., Altesor, A., Rodríguez, C. y Oesterheld, M. (2004). “Cambios estructurales y funcionales asociados al pastoreo en los pastizales del Río de la Plata", Reunión del Grupo técnico regional del Cono Sur en mejoramiento y utilización de los recursos forrajeros del área tropical y subtropical, Grupo Campos, núm. 20, pp. 53-60.

Perelman, S., Burkart, S. y León, C. (2003). "The role of a native tussock grass (Paspalum quadrifarium Lam.) in structuring plant communities in the Flooding Pampa grasslands, Argentina", Biodiversity and Conservation, no. 12, pp. 225238.

Perelman, S., Batista, W. y León, C. (2005). "El estudio de la heterogeneidad de la vegetación. Fitosociología y técnicas relacionadas", in Oesterheld, M., M.R. Aguiar, C.M. Ghersa and J.M. Paruelo (ed.), La heterogeneidad de la vegetación de los agroecosistemas. Un homenaje a Rolando León, Editorial Facultad de Agronomía, Buenos Aires, pp. 321-350.

Pontius, R., Shusas, E. y McEachern, M. (2004). "Detecting important categorical land changes while accounting for persistence", Agriculture, Ecosystems and Environment, núm. 101, pp. 251-268.

Price, K., Guo, X. y Stiles, J. (2002): “Optimal Landsat TM band combinations and vegetation indices for discrimination of six grassland types in eastern Kansas", International Journal of Remote Sensing, vol. 23, no. 23, pp. 5031-5042.

Rodríguez, A. y Jacobo, E. (2012), Manejo de pastizales naturales para una ganadería sustentable en la pampa deprimida: buenas prácticas para una ganadería 
sustentable de pastizal: kit de extensión para las pampas y campos, Fundación Vida Silvestre Argentina, Aves Argentinas, Buenos Aires.

Sala, O. y Paruelo, J. (1997). "Ecosystems services in grasslands", in G.C. Daily (ed.), Nature's Services: Societal Dependence on Natural Ecosystems, Washington, D.C., Island Press, pp. 237-251.

Schulz, J., Cayuela, L., Echeverría, C., Salas, J. y Rey Benayas, J. (2010). "Monitoring land cover change of the dryland forest landscape of Central Chile (19752008)", Applied Geography, no. 30, pp. 436-447.

Shi, Y., Xiao, J., Shen, Y. y Yamaguchi, Y. (2012). "Quantifying the spatial differences of landscapes change in the Hai River Basin, China, in the 1990s", International Journal of Remote Sensing, vol. 33, no. 14, pp. 4482-4501.

Soriano, A.; León, R.; Sala, O.; Lavado, R.; Deregibus, V.; Cahuépé, M.; Scaglia, O.; Velázquez, C. y Lemcoff, J. (1991). "Río de la Plata Grasslands", in R.T. Coupland (ed.), Natural Grasslands. Introduction and Western Hemisphere. Ecosystems of the World, Elsevier, New York, pp. 367-407.

Southworth, J., Munroe, D. y Nagendra, H. (2004). "Land cover change and landscape fragmentation - comparing the utility of continuous and discrete analyses for a western Honduras region", Agriculture, Ecosystems and environment, no. 101, pp. 185-205.

Tovar, C., Duivenvoorden, J., Sánchez-Vega, I. y Seijmonsbergen, C. (2012). "Recent changes in patch characteristics and plant communities in the Jalca grasslands of the Peruvian Andes", Biotropica, vol. 44, núm. 3, pp. 312-330.

Tricart, J. (1973). Geomorfología de la Pampa Deprimida, Colección Científica XII, INTA, Buenos Aires.

Tsakoumagkos, P. (2003). "Problemáticas ambientales y estudios sociales", en Bendini, M.; Cavalcanti, S.; Murmís, M.; Tsakoumagkos, P. (eds.), El campo en la sociología actual. Una perspectiva latinoamericana, Ediciones La Colmena, Buenos Aires, pp. 129-156.

Vecchio, M.; Golluscio, R. y Cordero, M. (2008). "Cálculo de la receptividad ganadera a escala de potrero en pastizales de la Pampa Deprimida", Ecología Austral, núm. 18, pp. 213-222.

Vervoorst, F.B. (1967). Las comunidades vegetales de la Depresión del Salado (Provincia de Buenos Aires). La vegetación de la República Argentina, Buenos Aires, Serie Fitogeográfica, 7, INTA.

Wu, J. y Hobbs, R. (2002). "Key issues and research priorities in landscape ecology: An idiosyncratic synthesis", Landscape Ecology, no. 17, pp. 355-365. 